\title{
Methods of Reduction of Unevenness \\ of the Transfer Characteristic of the Shaper \\ of Analog Counting of Digital Synthesizers \\ of Signals with Uniform Sampling
}

\author{
Dmitry S. Viktorov and Ekaterina V. Plastinina* \\ Military Academy of Aero-Space Defense \\ named after the Marshal of Soviet Union G.K. Zhukov \\ 50 Zhigareva Str., Tver, 170100, Russia
}

Received 24.05.2018, received in revised form 22.09.2018, accepted 23.04.2019

Digital methods of decrease of nonuniformity of the transmitting characteristic of the shaper of analog counting of digital synthesizers of signals with the uniform sampling are offered. The second method can be used also for decrease of noise of digital-to-analog transformation. Decrease in nonuniformity of the transmitting characteristic of the shaper of analog counting leads to increase in probability of the exact detection and allowing ability of radar stations.

Keywords: unevenness of the transfer characteristic, digital synthesizer of signals, shaper of analog counting, analog key, digital proofreader.

Citation: Viktorov D.S., Plastinina E.V. Methods of reduction of unevenness of the transfer characteristic of the shaper of analog counting of digital synthesizers of signals with uniform sampling, J. Sib. Fed. Univ. Eng. technol., 2019, 12(6), 630-640. DOI: $10.17516 / 1999-494 X-0162$.

(C) Siberian Federal University. All rights reserved

This work is licensed under a Creative Commons Attribution-NonCommercial 4.0 International License (CC BY-NC 4.0).

* Corresponding author E-mail address: viktorov.dmitry@yandex.ru, plastinina77@yandex.ru 


\title{
Методы уменышения неравномерности
}

\section{передаточной характеристики формирователя \\ аналоговых отсчетов цифровых синтезаторов сигналов \\ с равномерной дискретизацией}

\author{
Д.С. Викторов, Е.В. Пластинина \\ Военная академия воздушно-космической обороны \\ им. Маршала Советского Союза Г.К. Жукова \\ Россия, 170100, Тверь, ул. Жигарева, 50
}

$\overline{\text { Предложены иифровые методы уменьшения неравномерности передаточной характеристики }}$ формирователя аналоговых отсчетов иифровых синтезаторов сигналов с равномерной дискретизацией. Второй метод можно использовать и для уменьшения шумов иифроаналогового преобразования. Снижение неравномерности передаточной характеристики формирователя аналоговых отсчетов приводит к увеличению вероятности правильного обнаружения и разрешающей способности радиолокационных станций.

Ключевые слова: неравномерность передаточной характеристики, ицфровой синтезатор сигналов, формирователь аналоговых отсчетов, аналоговый ключ, ииифровой корректор.

Передаточная характеристика интерполятора цифрового синтезатора сигналов (ЦСС) имеет нелинейную амплитудно- и фазочастотные характеристики. Приемно-передающие тракты радиолокационных станций (РЛС) также имеют существенную нелинейность амплитудно- и фазочастотной характеристик. Это приводит к возникновению модуляционных искажений сигналов с угловой модуляцией (УМ) в виде паразитной нелинейной амплитудной и фазовой модуляций. При этом существенно уменьшается вероятность правильного обнаружения и разрешающая способность РЛС. Эти важнейшие параметры РЛС во многом зависят от искажений амплитуды основного импульса и уровня боковых лепестков сжатого сигнала на выходе устройства обработки РЛС.

В ЦСС с равномерной дискретизацией (РД) передаточная характеристика формирователя аналоговых отсчетов $(Ф А О) K_{\mathrm{O}}(\omega)$ имеет существенную неравномерность, которая может достигать нескольких десятков процентов. Это приводит к возникновению модуляционных искажений в виде паразитной амплитудной модуляции сигнала с УМ, которая, в общем случае, нежелательна и должна быть устранена. Величина максимальной среднеквадратической ошибки при формировании сигналов с УМ может достигать значения порядка $4 \cdot 10^{-2}$. Наиболее простой способ устранения неравномерности передаточной характеристики ФАО состоит в применении аналоговых корректирующих цепей [1], однако при этом не удается получить желаемую точность коррекции.

Значительно лучшие результаты показаны при использовании цифровых методов. Среди них можно выделить два основных.

Первый основан на включении дополнительного корректирующего звена, коэффициент передачи которого изменяется во времени обратно пропорционально значению коэффициента передачи ФАО $K_{\mathrm{O}}(\omega)$ в зависимости от мгновенной частоты синтезируемого сигнала с УМ: 


$$
K_{\kappa}(t)=1 / K_{0}[\omega(t)]=\frac{\omega(t) T_{\ni} / 2}{\sin \omega(t) T_{\ni} / 2},
$$

где $\omega(t)$ - значение мгновенной частоты формируемого сигнала с УМ.

Варианты технической реализации данного метода показаны на рис. 1, 2 и 3.

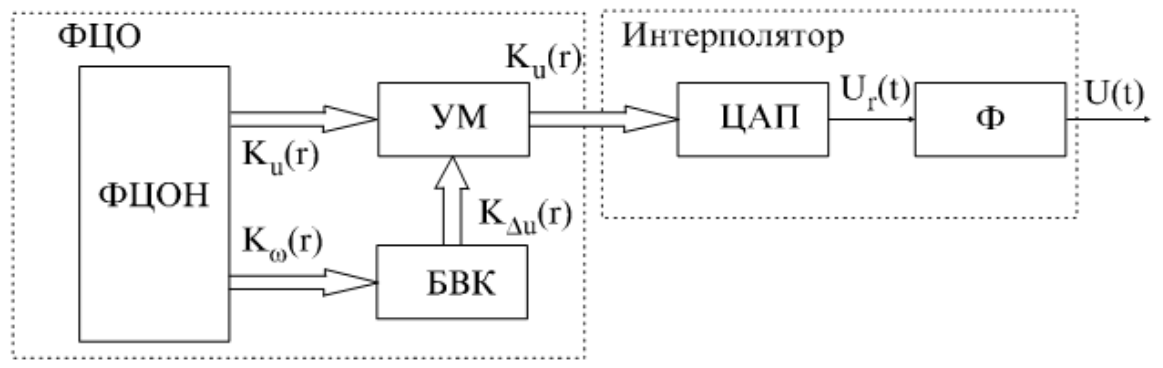

Рис. 1. Техническая реализация первого цифрового метода устранения неравномерности передаточной характеристики ФАО, вариант 1

Fig. 1. Technical realization of the first digital method of elimination of nonuniformity of the transmitting characteristic of the shaper of analog counting, option 1

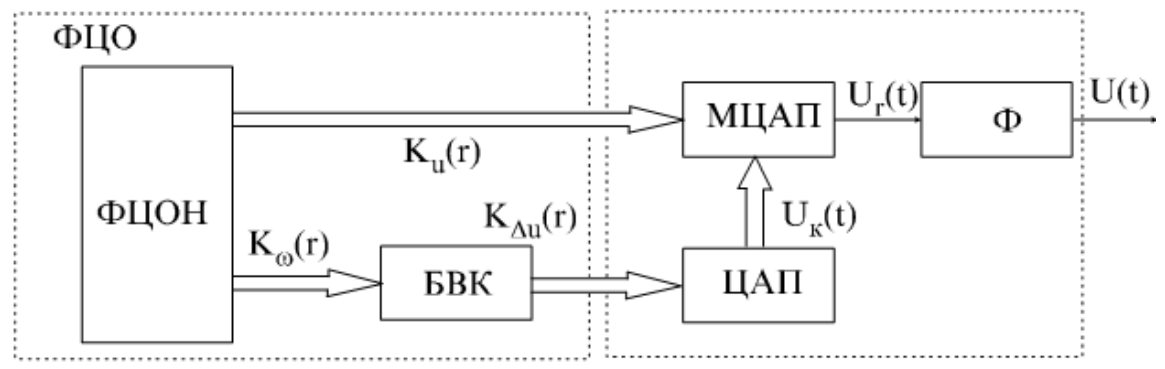

Рис. 2. Техническая реализация первого цифрового метода устранения неравномерности передаточной характеристики ФАО, вариант 2

Fig. 2. Technical realization of the first digital method of elimination of nonuniformity of the transmitting characteristic of the shaper of analog counting, option 2

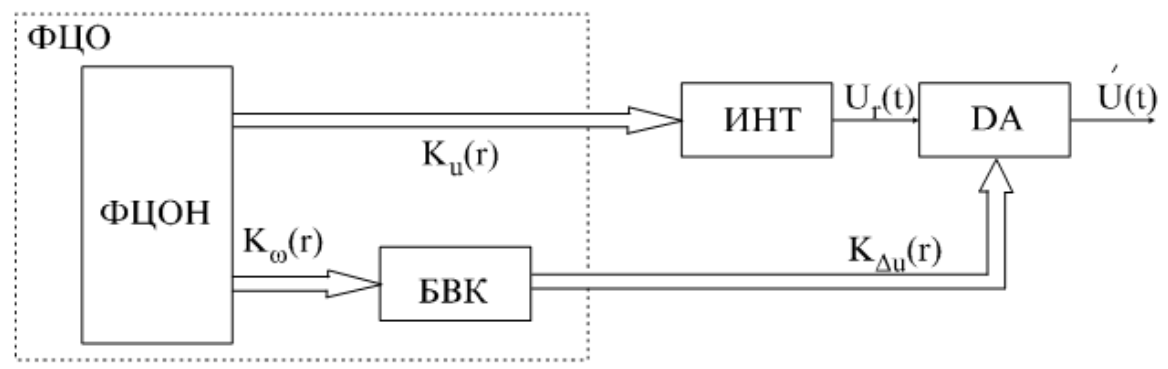

Рис. 3. Техническая реализация первого цифрового метода устранения неравномерности передаточной характеристики ФАО, вариант 3

Fig. 3. Technical realization of the first digital method of elimination of nonuniformity of the transmitting characteristic of the shaper of analog counting, option 3 
Основным узлом корректирующей цепи является блок вычисления кода (БВК), на вход которого с формирователя цифровых отсчетов (ФЦО) подается информация $K_{\omega}$ о мгновенной частоте синтезируемого сигнала с УМ. Вычисленный код коррекции $K_{\kappa}$ в первом варианте (рис. 1) подается на один вход умножителя включенного между ФЦО напряжения и цифроаналогового преобразователя (ЦАП). В этом случае производится коррекция цифровых отсчетов мгновенного напряжения формируемого сигнала с УМ. Во втором варианте (рис. 2) коды коррекции $K_{\kappa}$ преобразуются с помощью ЦАП в аналоговое напряжение $U_{k}(t)$, которое затем поступает на опорный вход множительного цифроаналогового преобразователя (МЦАП). В этом случае производится коррекция амплитуды аналоговых отсчетов мгновенного напряжения формируемого сигнала с УМ. В третьем случае (рис. 3) коды коррекции $K_{\kappa}$ подаются на управляющие входы дискретного аттенюатора (ДА), включенного на выходе интерполятора. Тогда производится коррекция амплитуды выходного аналогового сигнала. Если первые два варианта компенсации (рис. 1 и 2) могут использоваться только для цифрового синтезатора отсчетов напряжения (ЦСОН), то третий вариант (рис. 3) может быть применен как в ЦСОН, так и в цифровом синтезаторе отсчетов фазы (ЦСОФ).

При использовании умножителя (рис. 1) в качестве корректирующего звена приходится расширять динамический диапазон ЦАП путем добавления одного разряда [2]. К тому же при большом количестве разрядов квантования амплитуды $\ell$ умножитель становится слишком сложным. Однако данный метод очень эффективно можно использовать в ЦСОН табличного типа. В этом случае в запоминающее устройство ЦСС табличного типа необходимо записывать предварительно скорректированные в соответствии с (1) отсчеты напряжения.

Второй метод основан на непосредственном изменении передаточной характеристики ФАО в зависимости от значения мгновенной частоты синтезируемого сигнала с УМ [4]. Структурная схема ЦСОН, реализующая данный метод, приведена на рис. 4. ФАО данного ЦСС помимо ЦАП включает в себя преобразователь код-время (ПКВ) и аналоговый ключ (АК). Принцип работы такого ЦСС заключается в следующем. На выходе ЦАП формируются аналоговые отсчеты напряжения формируемого сигнала с УМ, длительность которых равна тактовому интервалу $T_{\text {э. }}$

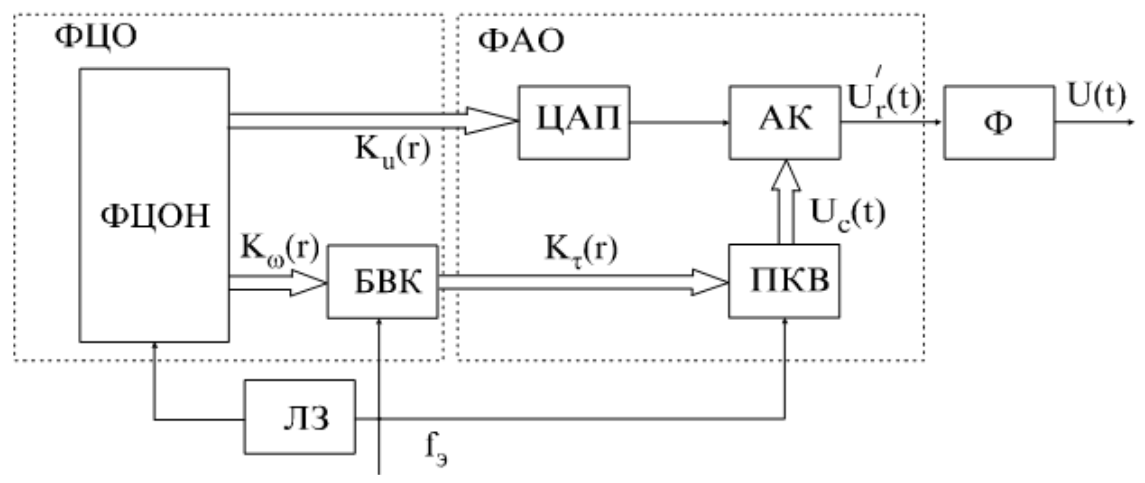

Рис. 4. Техническая реализация второго цифрового метода устранения неравномерности передаточной характеристики ФАО

Fig. 4. Technical realization of the second digital method of elimination of nonuniformity of the transmitting characteristic of the shaper of analog counting

$$
-633-
$$


С помощью АК, который открывается на короткое время $\tau$ импульсами, поступающими с ПКВ, формируются короткие аналоговые отсчеты формируемого сигнала длительностью $\tau$. Передаточная характеристика ФАО здесь определяется выражением

$$
K_{\tau}(\omega)=\frac{\tau}{T_{\ni}} \frac{\sin \omega \tau / 2}{\omega \tau / 2} e^{-j \omega \tau / 2}
$$

Поскольку длительность выборок на выходе АК меньше тактового интервала, передаточная характеристика модернизированного ФАО $K_{\tau}(\omega)$ уже первоначально имеет меньшую неравномерность по сравнению с передаточной характеристикой $K_{0}(\omega)$ прежнего ФАО. При изменении длительности аналоговых отсчетов передаточная характеристика ФАО также изменяется. На нижней частоте $f_{u}$ рабочего диапазона ЦСС длительность формируемых аналоговых отсчетов $\tau_{н}$ минимальная. С повышением значения формируемой частоты длительность аналоговых отсчетов увеличивается и достигает максимального значения $\tau_{8}$ на верхней частоте $f_{6}$ рабочего диапазона частот ЦСС. При этом длительность аналоговых отсчетов изменяется таким образом, чтобы значение передаточной характеристики ФАО было бы постоянным во всем рабочем диапазоне частот. Максимальная длительность аналоговых отсчетов выбирается из условия получения максимального значения передаточной характеристики ФАО на верхней частоте рабочего диапазона частот ЦСС. Данному условию соответствует длительность выборок $\tau_{\beta}=1 / 2$, и значение передаточной характеристики ФАО соответствует при этом $K_{\tau}\left(\omega_{6}\right)=1 / \pi T_{z} f_{6}=f_{T} / \pi f_{6}$. Длительность аналоговых отсчетов для других значений частот рабочего диапазона ЦСС находят из условия $K_{\tau}(\omega)=K_{\tau}\left(\omega_{\varepsilon}\right)$ и определяют из выражения [4]

$$
\tau(\omega)=\frac{2 \arcsin \left(\omega / \omega_{\%_{0}}\right)}{\omega}
$$

Блок вычисления коррекции может быть выполнен различным образом. Более предпочтительно выполнение БВК в виде постоянного запоминающего устройства, в котором хранятся коды длительности аналоговых отсчетов различных значений частоты. Принцип построения преобразователей код-время достаточно полно описан в [5].

Реальные ЦАП имеют конечное время установления выходного тока (напряжения). Кроме того, при цифроаналоговом преобразовании возникают шумовые выбросы из-за различия времени включения и выключения разрядов ЦАП [6, 7]. В качестве примера на рис. 5a-8a приведены осциллограммы реальных сигналов на выходе ЦАП при формировании гармонического колебания с частотой, кратной $f_{T} / 16$. На рис. 5б-8б приведены спектрограммы данных сигналов. Видно, что наличие шумов цифроаналогового преобразования приводит к появлению дополнительных искажений. Причем эти искажения имеют частоты, кратные в данном случае $f_{\kappa}=\kappa \Delta f$, где $\Delta f=f_{T} / 16, \kappa=0,1,2, \ldots, 15$. Амплитуда шумовых составляющих спектра в общем случае неравномерна и нелинейно зависит от кода синтезируемой частоты. Существенное уменьшение шумов цифроаналогового преобразования можно достичь, если на выход ЦАП поставить стробирующую схему [8]. Аналоговый ключ (рис. 4) можно одновременно использовать не только для уменьшения неравномерности передаточной характеристики ФАО, но и для стробирования шумов цифроаналогового преобразования. Синхронизацию ФЦО (рис. 4) необходимо 
осуществлять тактовыми импульсами, задержанными в линии задержки (ЛЗ) на длительность аналоговых отсчетов. При этом к моменту открывания аналогового ключа (рис. 58-8в) переходные процессы в ЦАП заканчиваются, тем самым на выходе ФАО устраняются шумы цифроаналогового преобразования. На рис. 5z-8г представлены спектрограммы сигнала на выходе аналогового ключа.

Использование аналогового ключа для стробирования шумов цифроаналогового преобразования позволяет существенно их уменьшить.

Выбросы на вершине аналоговых отсчетов (рис. 5в-8в; 5z-8г) обусловлены просачиванием управляющего напряжения аналогового ключа, неидентичностью диодов и перезарядом паразитных емкостей аналогового ключа.

Однако эти выбросы идентичны для каждого аналогового отсчета и не зависят от его амплитуды. Вследствие этого они порождают паразитные составляющие, частота которых кратна частоте $f_{T}, 2 f_{T}$ и т.д. (рис. 5z-8г). Эти паразитные составляющие находятся вне рабочего диапазона ЦСС и могут быть легко отфильтрованы.

Данный метод можно использовать также и для стабилизации передаточной характеристики ФАО цифровых синтезаторов отсчетов фазы. В этом случае помимо стабилизации выходного напряжения ЦСС можно также устранить переходные процессы дискретных фазовращателей.

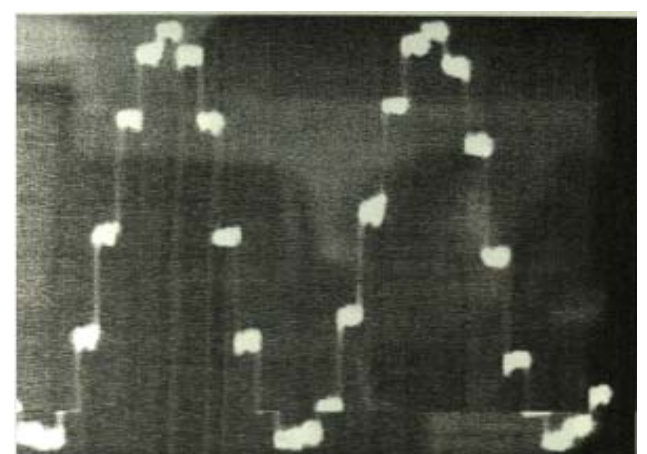

a)

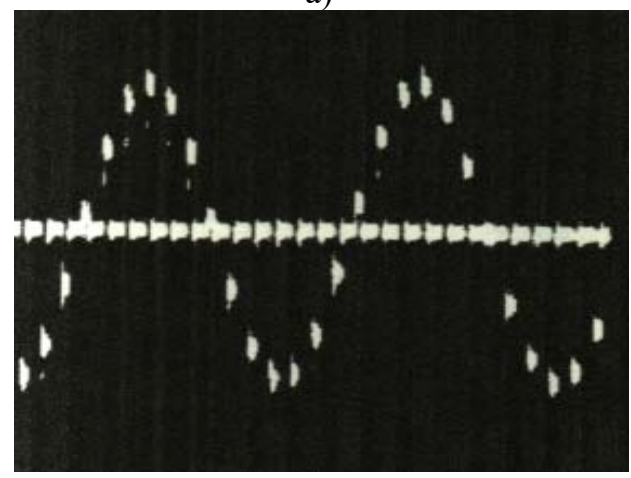

в)

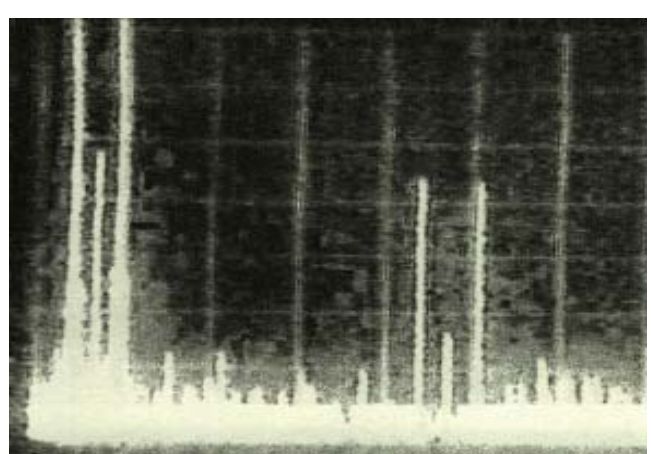

б)

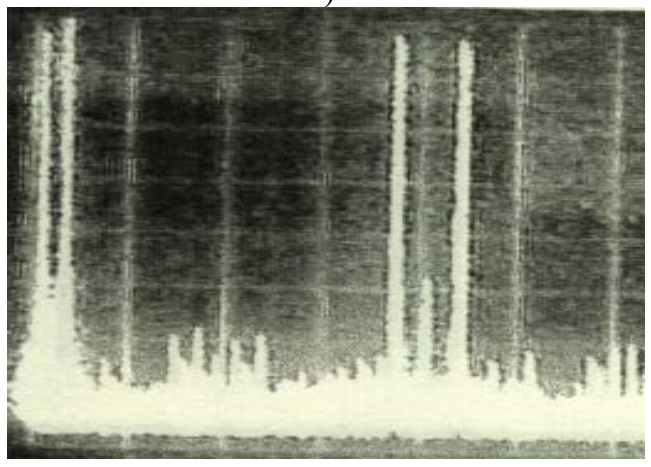

г)

Рис. 5. Осциллограммы и спектрограммы реальных сигналов на выходе ЦАП (а, б); на выходе АК (в, г)

Fig. 5. Oscillograms and spectrograms of actual signals at the exit of the digital-to-analog converter (a, b); at the exit of an analog key (c, d) 

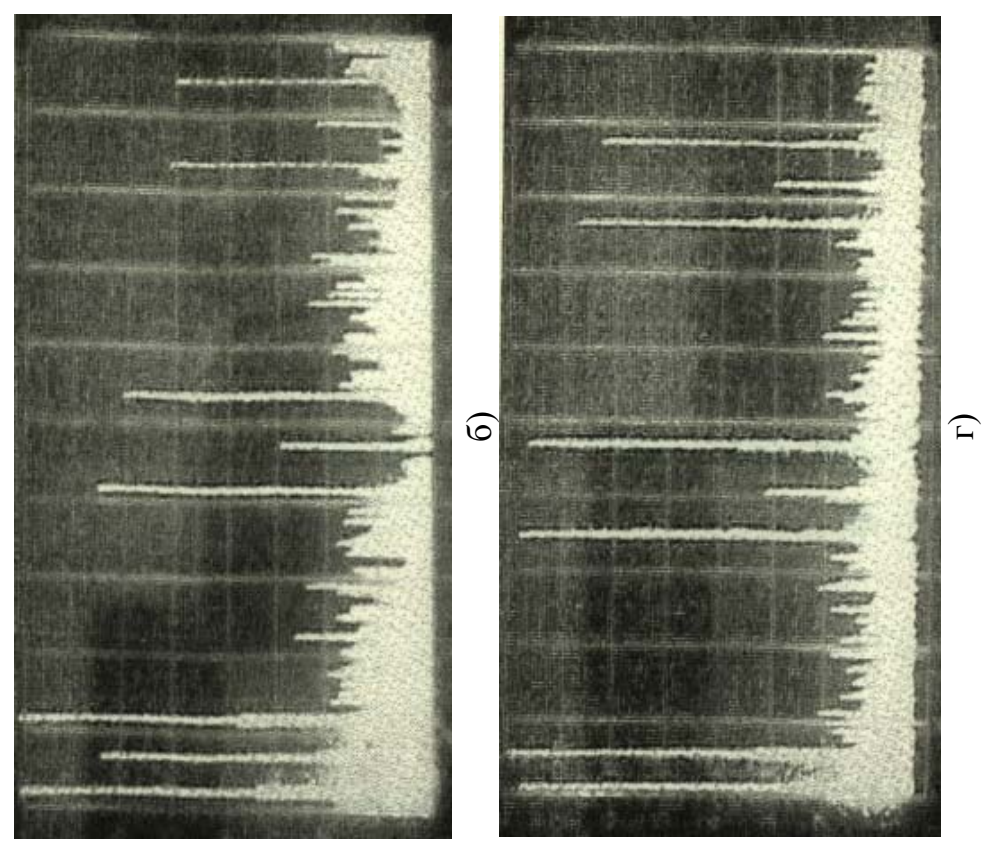

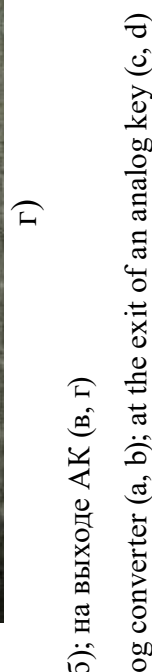
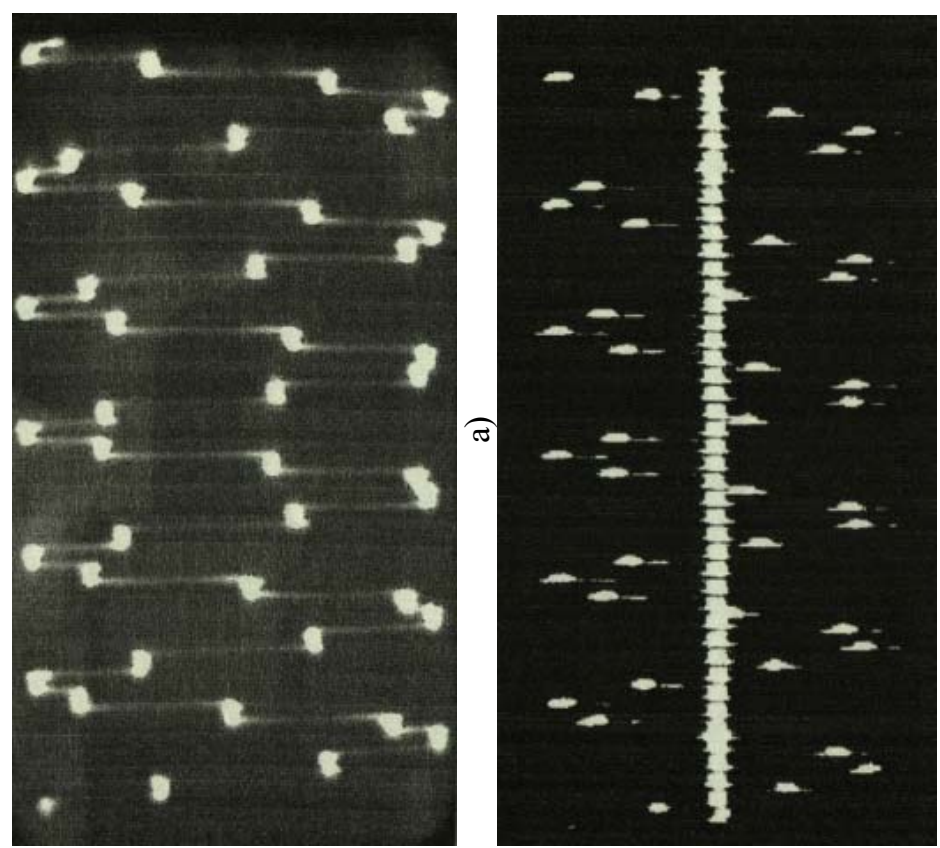

6ิ

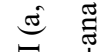

$\Xi \frac{1}{1}$

兽

究

氶 艺

舫

焉

5

范

๓)

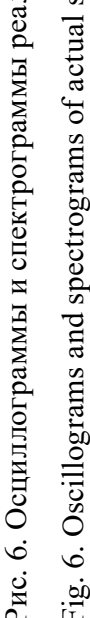




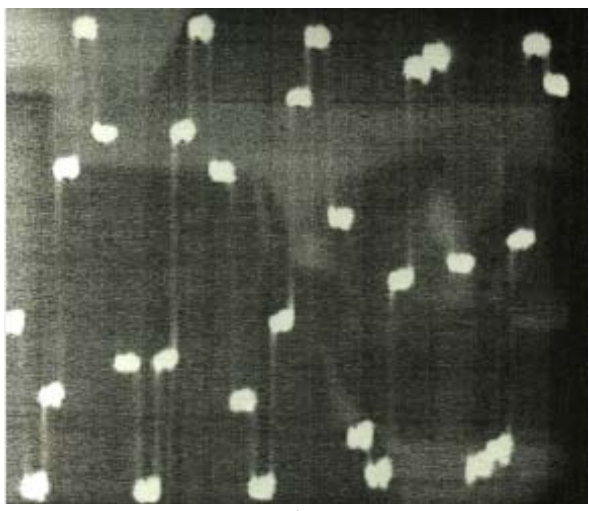

a)

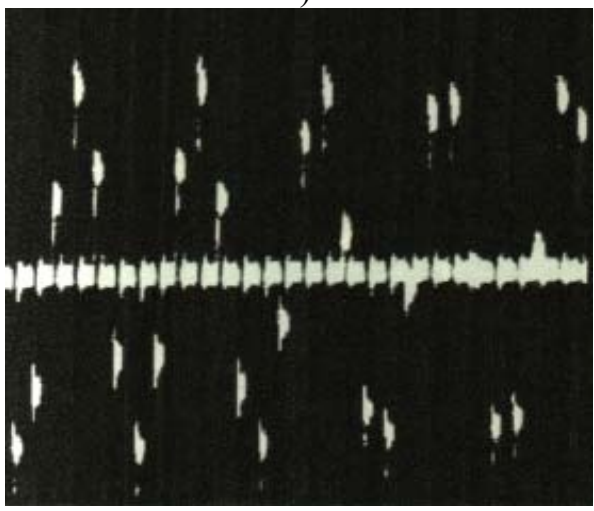

B)

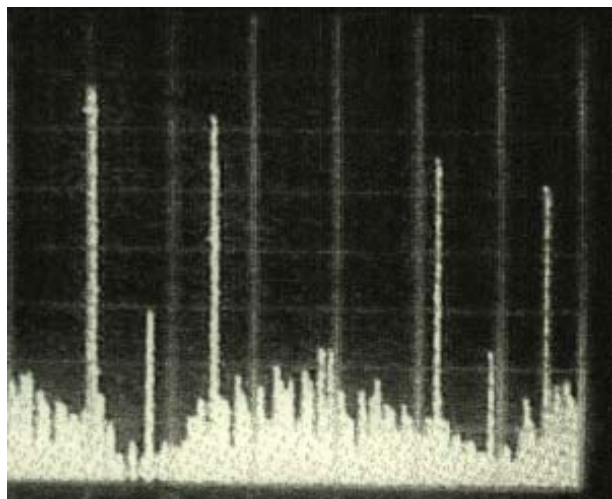

б)

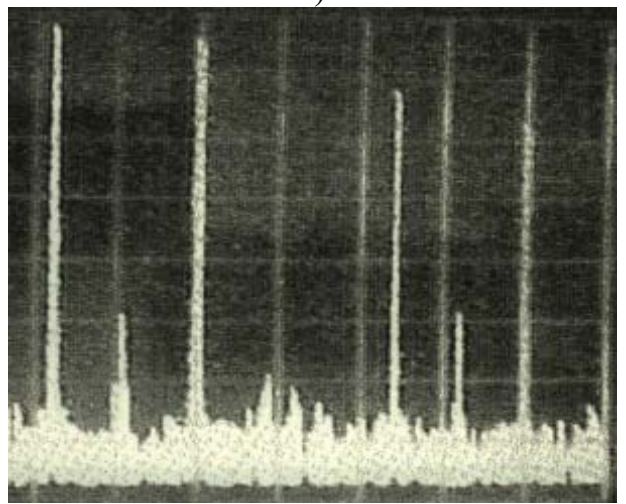

г)

Рис. 7. Осциллограммы и спектрограммы реальных сигналов на выходе ЦАП (а, б); на выходе АК (в, г)

Fig. 7. Oscillograms and spectrograms of actual signals at the exit of the digital-to-analog converter ( $a, b)$; at the exit of an analog key $(c, d)$

На рис. $9 a$ и $9 б$ приведены соответственно осциллограммы и спектрограммы ЛЧМ-сигнала на выходе ЦАП, а на рис. 9в и $9 z-$ на выходе аналогового ключа. Видно, что с уменьшением длительности аналоговых отсчетов неравномерность передаточной характеристики уменьшается.

Уменьшение неравномерности передаточной характеристики формирователя аналоговых отсчетов ЦСС с РД можно производить двумя методами. Первый метод основан на включении дополнительного корректирующего звена, коэффициент передачи которого изменяется во времени обратно пропорционально значению коэффициента передачи ФАО в зависимости от мгновенной частоты синтезируемого сигнала с УМ. Он реализуется включением корректирующего элемента, на основе цифрового умножителя, множительного ЦАП или дискретного аттенюатора. Второй метод основан на непосредственном изменении передаточной характеристики ФАО в зависимости от значения мгновенной частоты синтезируемого сигнала с УМ. Он реализуется с помощью дополнительно включенного аналогового ключа, длительность включения которого определяется мгновенной частотой синтезируемого сигнала.

Для уменьшения искажений, обусловленных конечным временем установления выходного тока (напряжения) и шумовыми выбросами из-за различия времени включения и выключения 


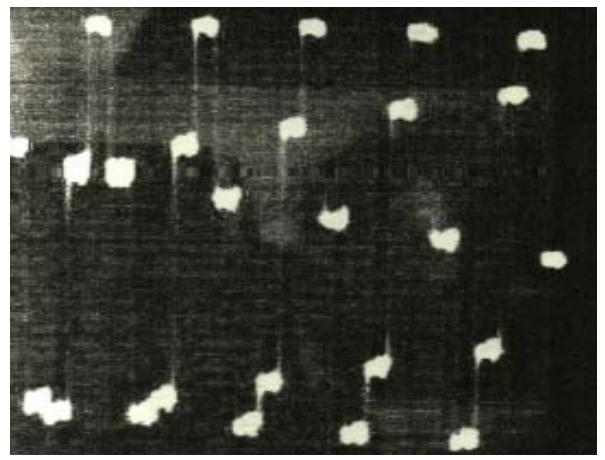

a)

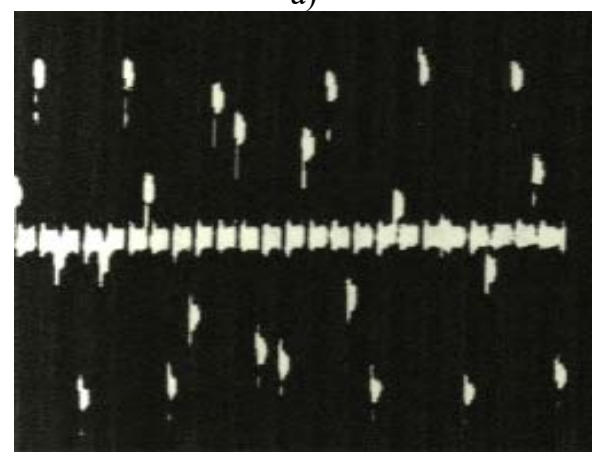

B)

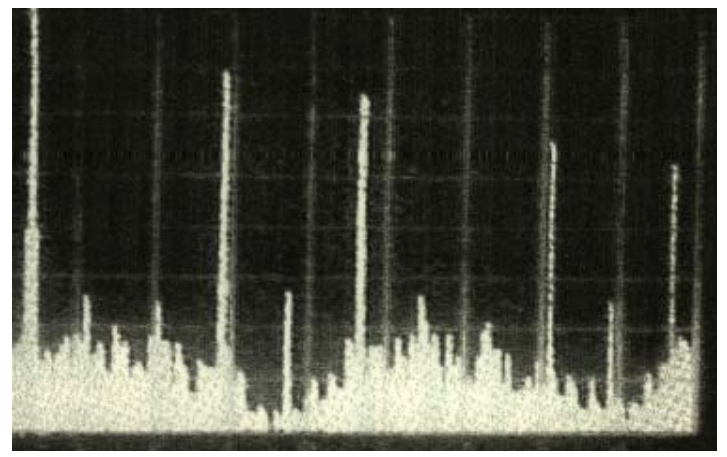

б)

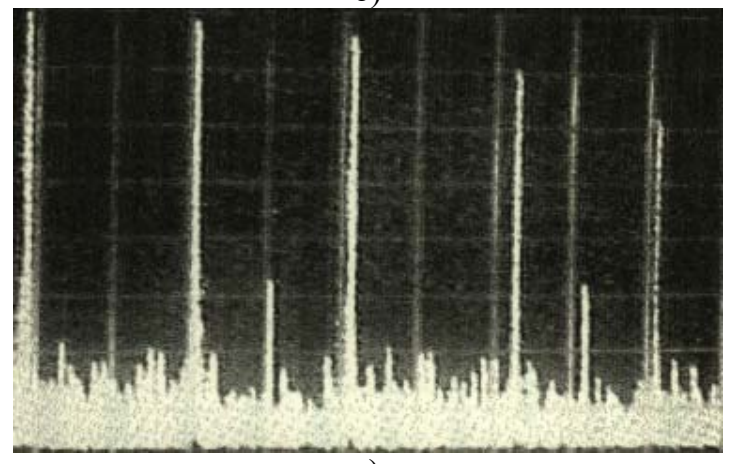

г)

Рис. 8. Осциллограммы и спектрограммы реальных сигналов на выходе ЦАП (а, б); на выходе АК (в, г)

Fig. 8. Oscillograms and spectrograms of actual signals at the exit of the digital-to-analog converter (a, b); at the exit of an analog key (c, d)

разрядов ЦАП в ЦСОН с РД, необходимо также использовать аналоговый ключ. Включение аналогового ключа должно производиться тогда, когда переходные процессы в ЦАП заканчиваются. При этом аналоговый ключ одновременно можно использовать как для уменьшения неравномерности передаточной характеристики ФАО, так и для уменьшения шумов цифроаналогового преобразования. 


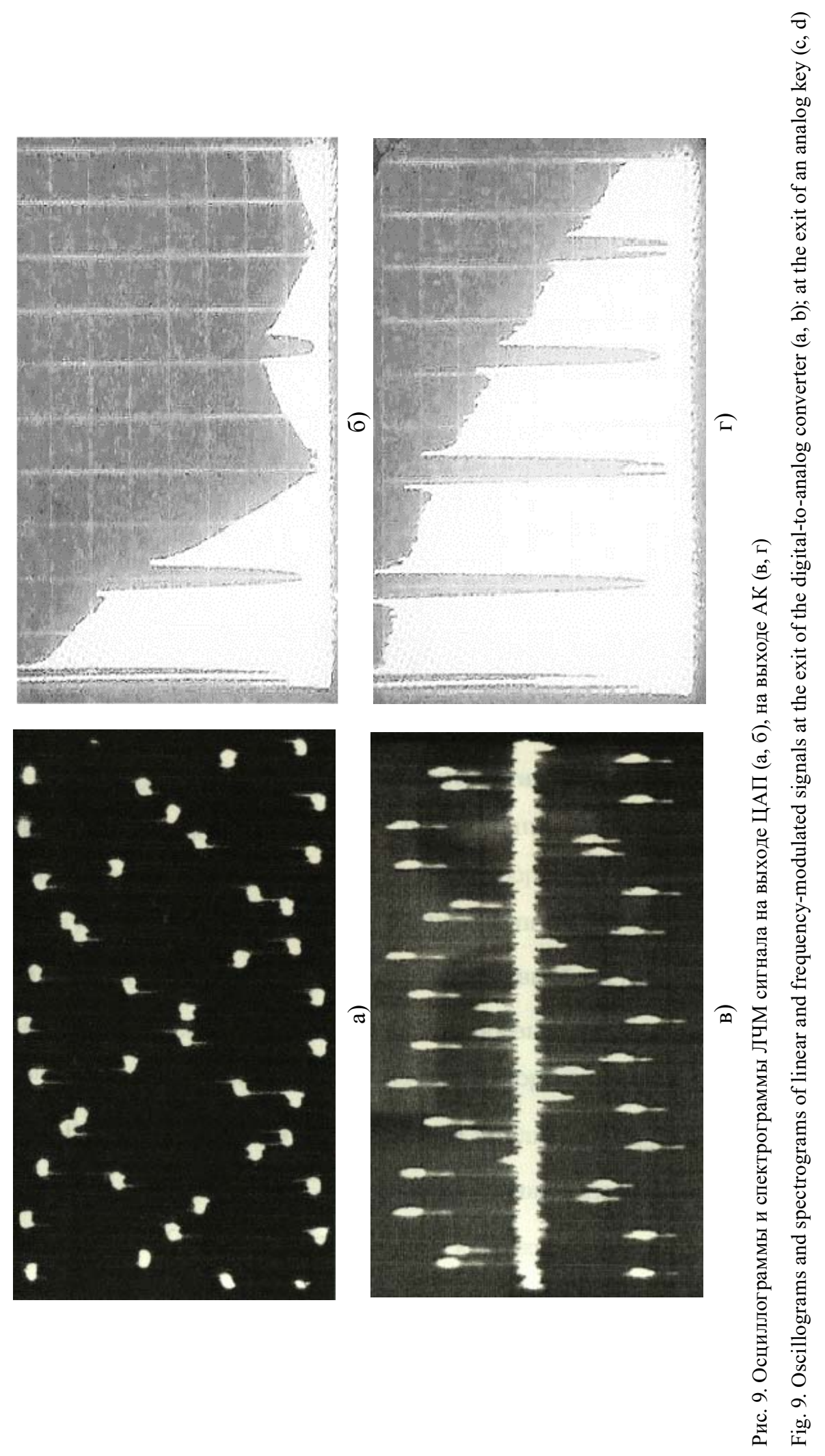




\section{Список литературы}

[1] Зааль Р. Справочник по расчету фильтров. М.: Радио и связь, 1983. 752c. [Zaal R. Reference book on calculation of filters. M.: Radio and communication, 1983. .752 p. (in Russian)].

[2] Раков И.А. Исследование и разработка методов повышения рабочих частот ииировых вычислительных синтезаторов сигналов. Автореф. дисс. ... канд. техн. наук. М.: ВЗЭИС, 1987. [Rakov I.A. Research and development of methods of increase in operating frequencies of digital computing synthesizers of signals. Avtoref. yew. PhD in Technological Sciences. M.: VZEIS, 1987 (in Russian)]

[3] Михайлов Н.А., Таджур С.М., Фомин А.В. Разработка иифровых синтезаторов ФМ и ЛЧМ сигналов. Заключ. отчет об ОКР “48Я6-Формирователь”. ВА ПВО, 1998. С.12-43. [Mikhaylov N.A., Tadzhur S.M., Fomin A.V. Development of digital synthesizers of fazo-and linearly frequency-modulated signals. Zaklyuch. report on ROC "48Ya6-Formirovatel". VA air defense, 1998. P. 12-43 (in Russian)].

[4] Hyghes A.S., Taylor S.A. Пат. №3792378 CШA. Digitalli controlled RF sweep generator. Кл. 331-178, заявл. 8.09.1971, опубл. 12.02.1979. [Hyghes A.S., Taylor S.A. Pat. No.3792378 USA. Digitalli controlled RF sweep generator. Registered 12 February 1979].

[5] Мелешко Е.А. Наносекундная электроника в экспериментальной физике. М.: Энергоатомиздат, 1987. 216c. [Meleshko E.A. Nanosecond electronics in experimental physics. M.: Energoatomizdat, 1987. 216 p. (in Russian)].

[6] Цифровая обработка сигналов. Справочник. Л.М. Гольденберг, Б.Д. Матюшин, М.Н. Поляк. М.: Радио и связь, 1985. 312c. [Digital processing of signals. Reference book. L.M. Goldenberg, B.D. Matyushin, M.N. Polyak. M.: Radio and communication, 1985. 312 p. (in Russian)].

[7] Бескин Л.Н. Цифровой синтезатор частоты. Радиотехника, 1980, 8, 63-66. [Beskin L.N. Digital synthesizer of frequency. Radio engineering, 1980, 8, 63-66 (in Russian)].

[8] Тирней, Рейдер, Голд. Цифровые синтезаторы частот. Зарубежная радиоэлектроника, 1972, 3, 54-74. [Tirney, Raider, Gold. Digital synthesizers of frequencies. Foreign radio electronics, 1972, 3, 54-74 (in Russian)]. 